\title{
Groups with fix-set quasi-order
}

\author{
Ian Hawthorn, Siva Manoharan, and Tim Stokes
}

\author{
Dedicated to Brian Davey on the occasion of his 65th birthday
}

\begin{abstract}
If $X$ is a set, the fix-set quasiorder on a group of permutations of $X$ is the quasiorder induced by containment of the fix-sets of elements of $S_{X}$. Axioms for such quasiorders on groups have previously been given. We generalise these to allow non-faithful group actions, the resulting abstract quasiorders being called fix-orders. We characterise the possible fix-orders on a given group $G$ in terms of certain families of subgroups of $G$. The special case in which the members of the defining family of subgroups are all normal is considered. Software is used to construct and analyse the lattices of fix-orders of many small finite groups.
\end{abstract}

\section{Introduction and summary of main results}

In any group of permutations $G \leq S_{X}$, in which composition is read left to right, one may define the fix-set quasi-order $\Subset$ by setting $g_{1} \Subset g_{2}$ if and only if $\operatorname{Fix}\left(g_{1}\right) \subseteq \operatorname{Fix}\left(g_{2}\right)$, where $\operatorname{Fix}(g)=\{x \in X \mid g(x)=x\}$. In [3], it was shown that $\Subset$ obeys the following laws: for all $g, h, k \in G$,

$$
\begin{aligned}
& 1 \Subset g \Rightarrow g=1, \\
& g \Subset g^{-1}, \\
& g \Subset h \& g \Subset k \Rightarrow g \Subset h k, \\
& g \Subset h \Leftrightarrow g^{k} \Subset h^{k},
\end{aligned}
$$

where $g^{k}=k g k^{-1}$ is the conjugate of $g$ by $k$. Conversely, it was shown that any group $G$ equipped with a quasi-order $\Subset$ satisfying these laws could be faithfully represented as a group of permutations in such a way that $\Subset$ corresponds to the fix-set quasi-order. So together with the group laws, the above laws axiomatize fix-set quasi-orders on permutation groups. Such axiomatizations of semigroups of functions equipped with various additional quasi-orders or other binary relations have been considered over the years by a number of authors: see the survey papers [5] and [6] (although much work has been done since).

Note that $\Subset$ is not expressible in the language of groups, so the addition of $\Subset$ to the group signature represents a proper enrichment of the language of permutations. Such an axiomatization means that every abstract property

Presented by G. McNulty.

Received October 17, 2013; accepted in final form February 13, 2014.

2010 Mathematics Subject Classification: Primary: 20F99; Secondary: 20D99, 20B40.

Key words and phrases: permutation group, quasi-order, lattice. 
of the fix-set quasi-order on groups of permutations can be formally deduced from these axioms without reference to concrete models. For example, the equational implication involving $f, g, h, k \in S_{X}$ of the form

$$
\forall x \in X: f(x)=g(x) \rightarrow h(x)=k(x)
$$

can be expressed in terms of the fix-set quasi-order as $f g^{-1} \Subset h k^{-1}$.

In [3], it was claimed that by dropping Law (1.1) above, the resulting axioms capture the fix-set quasi-order on groups acting (perhaps non-faithfully) on the right of a set $X$, defined as above but with

$$
\operatorname{Fix}(g)=\{x \in X \mid x \cdot g=x\} .
$$

We prove this explicitly here, using a slightly different construction that proves useful for our other goals.

We shall characterise fix-set quasi-orders on a group in terms of certain conjugate-closed families of subgroups. We single out the special case in which the subgroups are all normal; these correspond to examples embeddable in direct products of groups with a natural quasi-order. We show that the poset of all abstract fix-orders on a group forms a lattice, with sublattices consisting of the faithful fix-orders and the normal fix-orders. With the aid of some computer software, we determine these lattices for a large number of small finite groups.

\section{Definitions and basic properties}

A group with fix-order is a structure $(G, \Subset)$ in which $G$ is a group and $\Subset$ is a quasi-order on $G$ satisfying laws (1.2), (1.3) and (1.4); we also say that $\Subset$ is a fix-order on $G$. The group with fix-order $(G, \Subset)$ (and hence also the fix-order $\Subset)$ is said to be faithful if also (1.1) is satisfied.

Note that if $G$ is abelian, (1.4) is redundant. Abelian groups with fix-order generalise the abelian groups with a semilattice-valued norm-like operator considered in [1] where they were called modal abelian groups.

Every non-trivial group $G$ has at least two fix-orders: the full relation, in which $a \Subset b$ for $a, b \in G$; and the binary fix-order, in which $a \Subset 1$ for all $a \in G$, with $a \Subset b$ for all $a \neq 1$ and $b \neq 1$. The binary fix-order is obviously the largest faithful fix-order on $G$.

If $(G, \Subset)$ is a group with fix-order, then so is $(H, \Subset)$ where $H$ is any subgroup of $G$. There is also an obvious definition for the direct product of groups with fix-order. Indeed the class of groups with fix-order is a quasivariety in the sense of model theory, hence closed under substructures, direct products and ultraproducts.

It is routine to check that any group acting on a set is a group with fixorder under the fix-set quasi-order, and is faithful if and only if the group acts faithfully. Next we prove the converse, namely that every (faithful) group with fix-order arises in this way. This was proved in a far more general setting 
in [3], but only in the faithful case, and in any case, the group proof is rather simpler and we give it here for completeness.

We say a subgroup $S$ of a group with fix-order $(G, \Subset)$ is up-closed if $a \in S$ and $a \Subset b$ imply $b \in S$. Let $\mathcal{S}_{\Subset}(G)$ be the collection of all up-closed subgroups of $G$.

Let $(G, \Subset)$ be a group with fix-order. Then for every $a \in G$, define

$$
S_{a}=\{g \in G \mid a \Subset g\} .
$$

Lemma 2.1. In a group with fix-order $(G, \Subset), S_{a}$ is an up-closed subgroup for all $a \in G$.

Proof. If $x, y \in S_{a}$, then $a \Subset x$ and $a \Subset y$, so $a \Subset x y$ by (1.3), and also $a \Subset x^{-1}$ by (1.2) and transitivity, so $x y, x^{-1} \in S_{a}$. Obviously, $S_{a}$ is up-closed, so $S_{a} \in \mathcal{S}_{\Subset}(G)$.

Theorem 2.2. Every group with fix-order is isomorphic to a group acting on a set equipped with the fix-set quasi-order, and is faithful if and only if the group action is faithful.

Proof. Let $a, b \in G$. Note that if for all $S \in \mathcal{S}_{\Subset}(G)$ we have $a \in S$ implies $b \in S$, then choosing $S=S_{a}$ gives that $a \Subset b$; conversely, it is obvious that if $a \Subset b$, then $a \in S$ implies $b \in S$ for all $S \in \mathcal{S}_{\Subset}(G)$.

Now let $G$ act on the right cosets in $G / S$, where $S \in \mathcal{S}_{\Subset}(G)$, by multiplication in the usual way: $(S \cdot x) g:=S \cdot(x g)$. Of course, this is well defined. Do this for every $g \in G$ and then let $G^{*}=\prod_{S \in \mathcal{S}_{\in}(G)} G / S$ with $G$ acting on the right of $G^{*}$ in the obvious manner. Let $\preceq$ be the associated fix-set quasi-order on $G$. The following are equivalent for all $g_{1}, g_{2} \in G$ :

(1) $g_{1} \preceq g_{2}$;

(2) for all $S \in \mathcal{S}_{\Subset}(G)$ and all $x \in G,(S \cdot x) g_{1}=S \cdot x \rightarrow(S \cdot x) g_{2}=S \cdot x$;

(3) for all $S \in \mathcal{S}_{\Subset}(G)$ and all $x \in G, S \cdot\left(x g_{1}\right)=S \cdot x \rightarrow S \cdot\left(x g_{2}\right)=S \cdot x$;

(4) for all $S \in \mathcal{S}_{\Subset}(G)$ and all $x \in G, x g_{1} x^{-1} \in S \rightarrow x g_{2} x^{-1} \in S$;

(5) for all $x \in G, g_{1}^{x} \Subset g_{2}^{x}$;

(6) $g_{1} \Subset g_{2}$.

Hence, $\preceq$ and $\Subset$ coincide. It is obvious that a group action on a set is faithful if and only if (1.1) is satisfied by the fix-set quasi-order.

So, every group with fix-order is isomorphic to a group acting on a set equipped with the fix-set quasi-order, and is faithful if and only if the group action is faithful.

Every non-faithful group with fix-order can be turned into a faithful one by factoring out the elements equivalent to unity.

Proposition 2.3. For $(G, \Subset)$ a group with fix-order, $S_{1}=\{g \in G \mid 1 \Subset g\}$ is a normal subgroup of $G$, and $\Subset_{1}$ on $G_{1}=G / S_{1}$, defined by setting

$$
S_{1} \cdot a \Subset_{1} S_{1} \cdot b \text { if and only if } a \Subset b,
$$

is well defined and is a faithful fix-order on $G_{1}$. 
Proof. $S_{1}$ is a subgroup by Lemma 2.1. If $a \in S_{1}$, then $1 \Subset a$, so for all $g \in G$, we have $1=g 1 g^{-1} \Subset g a g^{-1}$ and $g a g^{-1} \in S_{1}$. If $a \Subset b$, then for any $g, h \in S_{1}$, we have $a g \Subset a g g^{-1}=a \Subset b \Subset b h$, so $a g \Subset b h$; hence, $\Subset_{1}$ on $G_{1}$ is well defined. If $S_{1} \cdot 1 \Subset_{1} S_{1} \cdot g$, then $1 \Subset g$, and so $g \in S_{1}$. Hence, $S_{1} \cdot g=S_{1} \cdot 1$, and so $\Subset_{1}$ is faithful.

Suppose $G$ is a group with $N$ normal in $G$, and suppose $(G / N, \Subset)$ is a group with fix-order. On $G$, define $\Subset^{N}$ by setting $a \Subset^{N} b$ if and only if $a N \Subset b N$. It is straightforward to see that $\Subset^{N}$ is a fix-order on $G$, faithful if and only if $N=\{1\}$ and $\Subset$ is faithful, and we call it the extension of $\Subset$ by $N$.

Corollary 2.4. Every non-faithful fix-order on a group is an extension of a faithful fix-order on some quotient $G / N$ of $G$ by $N$.

Because every non-trivial group possesses at least one faithful fix-order, namely the binary fix-order, we obtain the following.

Corollary 2.5. Every fix-order except the full relation on a non-trivial group is faithful if and only if the group is simple.

\section{Fix-orders and families of subgroups}

Next, we show that the fix-orders on a group correspond to certain families of subgroups.

Let $G$ be a group, with $\mathcal{S}$ a non-empty collection of subgroups of $G$. We say $\mathcal{S}$ is conjugate-closed if for all $g \in G$ and $S \in \mathcal{S}, g S g^{-1} \in \mathcal{S}$. For any conjugate-closed collection of subgroups $\mathcal{S}$ of the group $G$, define the relation $\Subset_{\mathcal{S}}$ by setting, for all $g_{1}, g_{2} \in S$,

$$
g_{1} \Subset_{\mathcal{S}} g_{2} \Leftrightarrow\left(\forall S \in \mathcal{S}: g_{1} \in S \rightarrow g_{2} \in S\right) .
$$

If $\mathcal{S}$ has a single element $S$, we write $\Subset_{S}$ rather than $\Subset_{\{S\}}$.

Proposition 3.1. With $\Subset_{\mathcal{S}}$ as just defined, $\left(G, \Subset_{\mathcal{S}}\right)$ is a group with fix-order, which is faithful if and only if $\cap \mathcal{S}=\{1\}$.

Proof. That $\Subset_{\mathcal{S}}$ is a quasi-order is easily seen. Laws (1.2) and (1.3) follow from the fact that $\mathcal{S}$ consists of subgroups. For the final law, suppose $g_{1} \Subset_{\mathcal{S}} g_{2}$. Then supposing that $u g_{1} u^{-1} \in S$, we have that $g_{1} \in u^{-1} S u \in \mathcal{S}$, and so $g_{2} \in u^{-1} S u$, so $u g_{2} u^{-1} \in S$; hence $u g_{1} u^{-1} \Subset_{\mathcal{S}} u g_{2} u^{-1}$. If $1 \Subset_{\mathcal{S}} g$, then $g \in \bigcap \mathcal{S}$, so $\Subset_{\mathcal{S}}$ is faithful if and only if $\bigcap \mathcal{S}=\{1\}$.

Obviously, if $\mathcal{S}$ and $\mathcal{T}$ are conjugate-closed families of subgroups on the group $G$, with $\mathcal{S} \subseteq \mathcal{T}$, then $\Subset_{\mathcal{T}} \subseteq \Subset_{\mathcal{S}}$. The converse fails as the following easy example shows.

Let $G$ be a non-trivial group, let $\mathcal{S}=\{\{1\}, G\}$ and $\mathcal{T}=\{\{1\}\}$. Then $\Subset_{\mathcal{T}} \subseteq \Subset_{\mathcal{S}}$; indeed, these two fix-orders both equal the binary fix-order (in which 1 is largest with all other elements related in both directions), yet $\mathcal{S} \subseteq \mathcal{T}$ fails. 
In fact, all fix-orders on a group arise from families of subgroups.

Proposition 3.2. Let $(G, \Subset)$ be a group with fix-order. Then the family of subgroups $\mathcal{S}_{\Subset}(G)$ is conjugate-closed, and $\Subset$ equals $\Subset_{\mathcal{S}_{\Subset}(G)}$.

Proof. Let $S \in \mathcal{S}_{\Subset}(G)$, with $g \in G$. Suppose $a, b \in G$ are such that $a \in g S g^{-1}$ and $a \Subset b$. So, $a=g s g^{-1}$ for some $s \in S$. Then $s=g^{-1} a g \Subset g^{-1} b g$ from (1.4), and so because $S$ is up-closed, $g^{-1} b g \in S$; hence, $b \in g S g^{-1}$. Thus, $\mathcal{S}_{\Subset}(G)$ is conjugate-closed. The second part follows easily from the proof of Theorem 2.2.

This shows that all groups with fix-order arise from conjugate-closed families of subgroups. Two distinct such families may give rise to the same fix-order $\Subset$, but $\mathcal{S}_{\Subset}(G)$ is easily seen to be the largest such family (since if $\mathcal{S}$ is a conjugateclosed family yielding $\Subset$, then each $H \in \mathcal{S}$ is up-closed with respect to $\Subset$, and hence belongs to $\mathcal{S}_{\Subset}(G)$ ). There is interest in characterising families of the form $\mathcal{S}_{\Subset}(G)$ without reference to fix-orders.

Proposition 3.3. If $\mathcal{S}$ is a conjugate-closed family of subgroups of the group $G$ with union $G$, then the subgroup $S$ of $G$ is up-closed relative to $\Subset_{\mathcal{S}}$ if and only if it is a union of intersections of elements of $\mathcal{S}$.

Proof. It is clear that if $S$ is up-closed, then it is the union of the subgroups $S_{a}=\left\{b \in G \mid a \Subset_{\mathcal{S}} b\right\}$, for $a \in S$; but it is easy to see that $S_{a}=$ $\bigcap\{T \in \mathcal{S} \mid a \in T\}$, which is a non-empty intersection since $\mathcal{S}$ has union $G$. Conversely, if $S$ is a union of intersections of subgroups in $\mathcal{S}$, then $S$ is easily seen to be up-closed.

The following notion has a ring-theoretic analog which was explored in [4], where rings of endomorphisms of abelian groups equipped with the quasi-order induced by kernel inclusion were axiomatized.

Definition 3.4. For a group $G$, we call the family of subgroups $\mathcal{S}$ ordercomplete if it satisfies the following conditions:

- $\mathcal{S}$ is closed under arbitrary intersections;

- $G \in \mathcal{S}$;

- if $S$ is a subgroup of $G$ that is a union of subgroups in $\mathcal{S}$, then $S \in \mathcal{S}$.

Proposition 3.5. A family of subgroups $\mathcal{S}$ of the group $G$ equals $\mathcal{S}_{\Subset}(G)$ for some fix-order $\Subset$ on $G$ if and only if $\mathcal{S}$ is conjugate-closed and order-complete.

Proof. Suppose $\mathcal{S}$ is conjugate-closed and order-complete. By Proposition 3.3, $S$ is up-closed under $\Subset_{\mathcal{S}}$ if and only if it is a union of intersections of elements of $\mathcal{S}$, that is, it is an element of $\mathcal{S}$. So $\mathcal{S}=\mathcal{S}_{\complement_{\mathcal{S}}}(G)$.

Conversely, suppose $\Subset$ is a fix-order on $G$. We saw in Proposition 3.2 that $\mathcal{S}_{\Subset}(G)$ is conjugate-closed. Moreover, $G$ itself is obviously up-closed, and so Proposition 3.3 applies to $\mathcal{S}_{\Subset}(G)$. Hence, any intersection of elements of $\mathcal{S}_{\Subset}(G)$ is up-closed, and so is any union which is a subgroup. So, $\mathcal{S}_{\Subset}(G)$ is order-complete. 
It follows that $\Subset_{\mathcal{F}}$, where $\mathcal{F}$ consists of all subgroups of $G$, is the smallest fix-order on $G$, and it is obviously faithful since $\{1\}$ is a subgroup.

\section{Normal fix-orders}

If $G$ is a group and $\mathcal{S}$ is a family of normal subgroups, then $\mathcal{S}$ is trivially conjugate-closed. In this case, it is easy to see that the additional law

$$
x \Subset x^{y}
$$

holds for $\Subset_{\mathcal{S}}$ since for a normal subgroup $N$ of $G$, if $x \in N$, then so is every conjugate $x^{y}$ of $x$.

Generalising, let us call a fix-order on a group normal if it satisfies (4.1). Of course, in an abelian group, every fix-order is normal. Any extension of a normal fix-order by a normal subgroup is obviously normal. The full and binary fix-orders on any non-trivial group are both normal.

The following result shows that this law axiomatizes examples arising from families of normal subgroups.

Proposition 4.1. Let $(G, \Subset)$ be a group with normal fix-order. Then $\mathcal{S}_{\Subset}(G)$ consists entirely of normal subgroups.

Proof. From the proof of Theorem 2.2, $S \in \mathcal{S}_{\Subset}(G)$ are subgroups. But for any $S \in \mathcal{S}_{\Subset}(G)$, if $x \in S$ then $x \Subset x^{g}$ and so $x^{g} \in S$, and so $S$ is normal.

The next result now follows easily from Proposition 3.5.

Proposition 4.2. A family of subgroups $\mathcal{S}$ of the group $G$ equals $\mathcal{S}_{\Subset}(G)$ for some normal fix-order $\Subset$ on $G$ if and only if $\mathcal{S}$ consists entirely of normal subgroups and is order-complete.

In the faithful case, another interpretation of the above results is as follows. Let $G_{i}, i \in \mathcal{I}$ be a family of groups and let $G=\Pi_{i \in \mathcal{I}} G_{i}$. Define the quasi-order $\preceq$ on $G$ by setting, for $\left(g_{i}\right),\left(h_{i}\right) \in G$,

$$
\left(g_{i}\right) \preceq\left(h_{i}\right) \text { if and only if } g_{i}=1 \rightarrow h_{i}=1 \text { for all } i \text {. }
$$

So, $\left(g_{i}\right) \preceq\left(h_{i}\right)$ means that $\left(h_{i}\right)$ has a "1" in at least the same places as $\left(g_{i}\right)$. We call $\preceq$ the coordinate quasi-order on the direct product of the groups $G_{i}$.

Any subgroup with fix-order of the group with fix-order $(G, \preceq)$ as just defined is of course also a group with faithful normal fix-order. Conversely, we have the following.

Proposition 4.3. Let $(G, \Subset)$ be a group with faithful normal fix-order. Then $G$ is embeddable in a direct product of groups equipped with its coordinate quasi-order.

Proof. Each $S \in \mathcal{S}_{\Subset}(G)$ is normal, so each $G / S$ is a group. Now, $\{1\} \in \mathcal{S}_{\Subset}(G)$, so $\bigcap_{S \in \mathcal{S}_{\Subset}(G)} S=\{1\}$ trivially. Hence, $G$ is a subdirect product of the $G / S$ and $\Subset$ on $G$ maps to the coordinate quasi-order on $\Pi_{S \in \mathcal{S}} G / S$ under the canonical embedding. 
The criterion for simplicity given in Corollary 2.5 turns out to be just one of several.

Corollary 4.4. The following are equivalent for the non-trivial group G.

(1) $G$ is simple.

(2) Every non-full fix-order on $G$ is faithful.

(3) Every non-full normal fix-order on $G$ is faithful.

(4) Every normal fix-order on $G$ is either full or binary.

Proof. $(1) \Rightarrow(2)$ : This follows from Corollary 2.5 .

$(2) \Rightarrow(3)$ : This is immediate.

$(3) \Rightarrow(1)$ : If $G$ is not simple, let $N$ be a proper non-trivial normal subgroup. Then $G / N$ is non-trivial and has a faithful normal fix-order (namely the binary fix-order, in which 1 is the unique largest element and $a \Subset b$ if $a, b \in G \backslash\{1\})$. Extend to a normal fix-order on $G$ which is not faithful.

$(1) \Leftrightarrow(4)$ : The normal fix-orders on $G$ correspond one-to-one with its ordercomplete families of normal subgroups. Clearly, $\mathcal{S}=\{G\}$ is the order-complete family corresponding to the full fix-order (in which $a \Subset b$ for all $a, b \in G$ ), and $\mathcal{T}=\{\{1\}, G\}$ is the order-complete family corresponding to the binary fixorder. These are the only order-complete families if and only if $G$ is simple (as otherwise there will be a bigger one).

\section{The lattice of fix-orders on a group}

As we have already seen, the fix-orders on a given group are in one-to-one correspondence with its conjugate-closed order-complete families of subgroups. We next extend this correspondence to the structure of the associated lattices. The results of this section have ring-theoretic analogs in [4].

Let $G$ be a non-trivial group, with $\mathcal{C}_{G}$ the collection of all conjugate-closed families of subgroups of $G$, and $\mathcal{F}_{G}$ the collection of all fix-orders on $G$. Define the mappings $f: \mathcal{C}_{G} \rightarrow \mathcal{F}_{G}$ and $g: \mathcal{F}_{G} \rightarrow \mathcal{C}_{G}$ by setting $f(\mathcal{S}):=\Subset_{\mathcal{S}}$ and $g(\Subset):=\mathcal{S}_{\Subset}(G)$, for all families $\mathcal{S}$ in $\mathcal{C}_{G}$ and fix-orders $\Subset$ in $\mathcal{F}_{G}$.

Proposition 5.1. The pair $(f, g)$ constitutes an antitone Galois connection between $\left(\mathcal{C}_{G}, \subseteq\right)$ and $\left(\mathcal{F}_{G}, \subseteq\right)$.

Proof. It is routine to check that $\Subset \subseteq \Subset_{\mathcal{S}}$ if and only if $\mathcal{S} \subseteq \mathcal{S}_{\Subset}(G)$, and hence $\Subset \subseteq f(\mathcal{S})$ if and only if $\mathcal{S} \subseteq g(\Subset)$, for all $\mathcal{S} \in \mathcal{C}_{G}$ and $\Subset \in \mathcal{F}_{G}$.

Under the Galois connection $(f, g)$, the closed sets in $\left(\mathcal{C}_{G}, \subseteq\right)$ are exactly the $\mathcal{S}_{\Subset}(G)$, the conjugate-closed order-complete subsets, by Proposition 3.5, whereas all elements of $\mathcal{F}_{G}$ are closed by Proposition 3.2. Both collections of closed sets are complete lattices in which meet is intersection, and are antiisomorphic. So in particular, for conjugate-closed order-complete families $\mathcal{S}$ and $\mathcal{T}$,

$$
\Subset_{\mathcal{S}} \vee \Subset_{\mathcal{T}}=\Subset_{\mathcal{S} \cap T}
$$


In the lattice $\mathcal{F}_{G}$, the largest element is the full quasi-order, which arises from the conjugate-closed order-complete family $\{G\}$, and the smallest is $\Subset_{\mathcal{H}}$, where $\mathcal{H}$ consists of all subgroups of $G$.

Because the binary fix-order on a non-trivial group is the largest faithful fix-order on it, the Galois connection $(f, g)$ of Proposition 5.1 restricts to one between the conjugate-closed families $\mathcal{S}$ of subgroups in $\mathcal{C}_{G}$ for which we have $\bigcap \mathcal{S}=\{1\}$, and the faithful fix-orders of $G$. The lattice of faithful fix-orders on $G$ is a complete sublattice of $\mathcal{F}_{G}$ (joins are as in $\mathcal{F}_{G}$ because the same is true in $\mathcal{C}_{G}$ - the intersection of two families with trivial intersection also has trivial intersection), having largest element the binary fix-order, and the same smallest element as $\mathcal{F}_{G}$.

Similarly, restricting to the normal fix-orders on $G$, we obtain a complete sublattice of $\mathcal{F}_{G}$ (joins are as in $\mathcal{F}_{G}$ because the intersection of two ordercomplete families of normal subgroups in $\mathcal{C}_{G}$ is one also), having largest element the full relation on $G$ (which is clearly normal), and smallest element $\Subset_{\mathcal{N}}$, where $\mathcal{N}$ consists of all normal subgroups of $G$.

Finally, restricting to the faithful normal fix-orders on $G$, we again obtain a complete sublattice of $\mathcal{F}_{G}$, having largest element the binary fix-order, and with smallest element as for the normal case just considered.

\section{The poset of inequivalent fix-orders}

Given a group $G$, we say that the fix-orders $\Subset_{1}, \Subset_{2}$ on $G$ are equivalent if there is a group automorphism $f: G \rightarrow G$ such that $f\left(\Subset_{1}\right)=\Subset_{2}$ (that is, $a \Subset_{1} b$ if and only if $\left.f(a) \Subset_{2} f(b)\right)$. So $\Subset_{1}, \Subset_{2}$ are equivalent if and only if the structures $\left(G, \Subset_{1}\right)$ and $\left(G, \Subset_{2}\right)$ are isomorphic. When this equivalence relation is applied to $\mathcal{F}_{G}$, the equivalence classes are a poset under inclusion in the obvious way.

Note that if $f$ is an inner automorphism on the group $G$, then there exists $g \in G$ such that $f(a)=g a g^{-1}$ for all $a \in G$. In that case, it is clear that any fix-order equivalent to $\Subset$ must equal $\Subset$, by law (1.4) for fix-orders. So such collapsing is only possible if $G$ possesses at least one outer automorphism.

\section{The lattices of fix-orders for small finite groups}

It is of interest to determine the lattice of fix-orders of some familiar finite groups, along with their sublattices of faithful and/or normal fix-orders. For a given small finite group, this can be done by determining all possible conjugate-closed families of its subgroups (adding in the zero intersection and/or normality assumption as required in order to obtain the various sublattices). Properties such as modularity and distributivity can also be determined.

To analyse large numbers of groups, an automated process was needed. We developed a software package built from the GAP system [2], which was 
used to extract some well-known information about a given group, such as the number of elements and subgroups of the group. The set of all fix-orders of the group $G$ considered was generated by first considering all the possible conjugate-closed families of subgroups of $G$. For each generated fix-order, its faithfulness and normality were tested by direct application of the definitions. Since each fix-order can be generated by more than one family of subgroups, the duplicate fix-orders were removed, resulting in a list of distinct fix-orders. (This was necessary because the order-completeness condition on families of subgroups was not used in this process.)

Once the set of all fix-orders on a group was known, the structure of the lattice of fix-orders for the group was tested for modularity and distributivity, and the meet- and join-irreducible elements identified. The lattice was then drawn as a directed acylic graph using a visualisation library, GraphViz, and the associated properties (faithfulness, meet-irreducibility etc.) were indicated on the graph by use of colour and borders of the nodes.

Finally, equivalent fix-orders in the sense of Section 6 were identified and collapsed together, resulting in a sometimes smaller poset. Examples showed that this poset is not always a lattice. These automorphism-collapsed posets were rendered alongside the original lattices.

Using this software, a dataset was created by running the software on many small groups. The dataset and the software are available at the following URL.

$$
\text { www.math.waikato.ac.nz/Staff/stokes/data/ }
$$

The generated dataset is presented in a web-based interactive format showing the properties of the lattice of fix-orders of each group in a tabular format, categorised by the type of group (for example, "Dihedral group") and sorted by the order of the group. The web-pages also allow all of the diagrams for any group to be viewed together, with filtering options to allow easier comparison of specific diagrams.

The dataset contains the complete descriptions of the lattices of fix-orders of all groups of order 15 or less, the first five alternating and symmetric groups, and most dihedral groups of order 126 or less. For each group, there are multiple diagrams, which correspond to the combination of properties of the fix-orders (faithfulness and normality) and the collapsing by automorphisms. In total, there are 12 diagrams per group and 120 groups in the dataset.

From the accumulated data, the following facts are readily observed:

- All fix-orders are normal in an abelian group. The smallest group having at least one non-normal fix-order is the symmetric group $S_{3}$ (the smallest nonabelian group), and conversely the smallest non-abelian group for which all fix-orders are normal is the quaternion group (of order 8).

- Every group has a non-faithful fix-order, namely the full relation, which is normal. The smallest groups with non-normal non-faithful fix-orders are of order twelve, namely the dicylic group Dic12 and the dihedral group $D_{12}$, each of which has two (out of their 20 and 98 fix-orders, respectively). 
- The group $A_{5}$ is noteworthy. It is the only simple group we have so far discovered that supports more than two fix-orders; it supports 20. Consistent with Corollary 4.4, the additional 18 fix-orders are all faithful and non-normal.

- All the groups considered here are such that if their lattice of fix-orders is modular, then it is distributive as well. The same applies to the sublattices of faithful, normal, and faithful normal fix-orders.

- The smallest group for which the lattice of all fix-orders is not modular is the Klein 4-group; this is also the smallest group for which the lattice of normal fix-orders is not modular. However, its lattice of faithful fix-orders is modular.

- The smallest groups for which the lattice of faithful fix-orders is not modular are the groups of order 8 other than the cyclic group; each of these also has its lattice of faithful normal fix-orders non-modular.

- Of the 28 groups of order 15 or less, precisely half have modular lattice of fix-orders, with no further ones having modular lattice of normal fix-orders, and 21 have modular lattice of faithful fix-orders. (A group of order 18, namely 18-1, has a non-modular lattice of fix-orders but a modular lattice of normal fix-orders.)

- The smallest group for which the lattice collapses non-trivially under automorphisms is the Klein 4-group.

- The example of 20-5, in which nodes 96 and 123 have no greatest lower bound (with both nodes 123 and 135 predecessors of both), shows that the poset of equivalence classes of fix-orders under automorphism equivalence need not be a lattice in general. The same example shows that this carries over to the faithful, normal, and faithful normal cases.

The dataset was used to infer patterns, such as the fact that all dihedral groups of order $2 p$, where $p>2$ is a prime, result in the same lattice of fixorders having six elements. This can easily be seen to be true in general by examining the conjugacy classes of subgroups of each of the groups.

Given the fourth bullet point above, we conjecture that distributivity is equivalent to modularity for all lattices of fix-orders and their various sublattices (at least for finite groups). This conjecture was tested on about 800 groups (all groups of order 255 or less having fewer than 2000 distinct fixorders), and no counterexamples were found.

Acknowledgement. The University of Waikato Summer Research Scholarship awarded to the second author made possible his significant contribution to this work, and we thank the university for funding it.

\section{REFERENCES}

[1] Fearnley-Sander, D., Stokes, T.: Equality algebras. Bull. Austral. Math. Soc. 56, 177-191 (1997)

[2] The GAP Group: GAP - Groups, Algorithms, and Programming, version 4.4.12 (2008). http://www.gap-system.org

[3] Stokes, T.: Axioms for function semigroups with agreement quasi-order. Algebra Universalis 66, 85-98 (2011) 
[4] Stokes, T.: Rings with kernel inclusion quasi-order. Algebra Universalis 70, 379-391 (2013)

[5] Schein, B.M.: Relation algebras and function semigroups. Semigroup Forum 1, 1-62 (1970)

[6] Schein, B.M.: Lectures on semigroups of transformations. Amer. Math. Soc. Transl. ser. 2. 113, 123-181 (1979)

\section{IAN HAWTHORN}

Department of Mathematics, The University of Waikato, Hamilton, New Zealand. e-mail: hawthorn@waikato.ac.nz

\section{Siva Manoharan}

Department of Computer Science, The University of Waikato, Hamilton, New Zealand. e-mail: smanoharan@outlook.com

Current address: 148 Galloway Street, Hamilton East, Hamilton, New Zealand.

\section{Tim Stokes}

Department of Mathematics, The University of Waikato, Hamilton, New Zealand. e-mail: stokes@waikato.ac.nz 